\title{
Condition monitoring and reliability of a resistance spot welding process
}

\author{
Matteo Strozzi, Marco Cocconcelli, Riccardo Rubini \\ Department of Sciences and Methods for Engineering, University of Modena and Reggio Emilia, Italy. \\ E-mail: matteo.strozzi@unimore.itriccardo.rubini@unimore.itmarco.cocconcelli@unimore.it \\ Gianmarco Genchi, Alessandro Zanella \\ CRF S.C.p.A., FCA Group, Strada Torino 50, 10043 Orbassano (Turin), Italy. \\ E-mail: gianmarco.genchi@fcagroup.com alessandro.zanella@crf.it
}

\begin{abstract}
The reliability of a resistance spot welding (RSW) process is studied monitoring the quality of the corresponding welding points. Each welding point is uniquely represented by a specific resistance characteristic curve over time. Five learning resistance characteristic curves, the good quality of the related welding points was experimentally verified by means of a non-destructive technique, are selected as a reference to check the quality of welding points related to different process resistance characteristic curves. A first estimate of the quality of the welding point is made comparing the corresponding process resistance characteristic curve with the learning maximum, minimum and average resistance characteristic curves. Both good quality and defective (glued or squeezed) welding points are observed. In order to more correctly identify the quality level of each welding point, two different parameters comparing the related process resistance characteristic curve with the learning average resistance characteristic curve are applied. First, the residual resistance, as the difference at each instant of time between the two resistance characteristic curves, is considered. Then, the Euclidean distance, as the geometric distance at each instant of time between the two resistance characteristic curves, is adopted. Finally, the trend of the quality of the welding points as their number increases for welding electrodes with a fixed number of dressings is investigated.
\end{abstract}

Keywords: Resistance spot welding, reliability, quality, resistance curves, residual resistance, Euclidean distance.

\section{Introduction}

RSW is a typology of welding that uses the heat produced by the Joule effect from the passage of electric current between two sheets pressed each against other by two copper electrodes housed on a clamping plier. The electric machine generates a high intensity electric current $(1000 \div 100000 \mathrm{~A})$ for a short time (fractions of one second), and the passage of electric current develops high amount of heat in a small area on the contact surface of the sheets (where the electrical resistance results maximum). The heat increases the temperature of the metal until it reaches its melting value, and the sequent solidification of the metal, which takes place while the electrodes are still pressed on the two sheets, leads to the formation of the welding point, see Mallick (2010). RSW process is widely applied for the production in series of metallic components due to very high precision level, operative velocity and automation degree, see works of Tumuluru (2010), Kashiyama and Murakawa (2013), Liu et al. (2010). In particular RSW process is used for thin aluminium or steel sheets in automobile field, such as for car frame, floor, side wall, doors and body, see Ambroziak and Korzeniowski (2010), Jou (2001), Manladan et al. (2015), Pouranvari and Marashi (2013).

A review of the current state of the art of RSW process monitoring can be found in the works of
Mallaradhya et al. (2018), Dhawale and Kulkarni (2017).

In the present work, RSW process is applied on a specific steel component located in the front part of the car body, having the task of absorbing both frontal and lateral impacts suffered by the car frame. The RSW process reliability is studied by considering the quality of the welding points corresponding to resistance characteristic curves that are continuously recorded by the acquisition system. The reference for welding point quality is given by five learning resistance characteristic curves experimentally verified by means of nondestructive ultrasonic method. Process resistance characteristic curves are first compared with the learning maximum, minimum, average resistance characteristic curves to obtain an initial estimate of the welding point quality. Then, by taking as a reference the former learning average resistance characteristic curve, the residual distance and the Euclidean distance of several process resistance characteristic curves are computed to derive the quality level of the related welding points.

The aim of the present work is the monitoring of the quality of a RSW process by analyzing the resistance characteristic curves corresponding to different welding points, in order to identify their defects and eventually the trend of the quality as their number increases using welding electrodes subjected to the same number of dressings. 


\section{Data analysis}

In this work, the RSW process is applied to weld the different sheets of a specific steel component located in the front part of a car body. The main task of this structural mechanical component is to absorb the energy generated by high-speed front and side collisions suffered by car chassis, so as to better protect the occupant of the car.

A large database is available consisting of a set of electric current, resistance, voltage and power characteristic curves, which were acquired using several sensors placed on RSW machine.

The full database is divided into learning and process database. The learning database consists of five characteristic curves, the good quality of the relative welding points was experimentally verified by means of non-destructive ultrasonic method. On the other hand, the process database comprises all the other characteristic curves, the quality level of the corresponding welding points will be monitored in this work by different data analysis techniques.

As the number of welded points increases, the contact surface of the electrodes becomes more worn and their diameter increases (the electrode tips have a truncated-conical shape). As a result, the quality of the welding points decreases (i.e., the diameter of the welding core and the current density at the welding point vary with respect to the reference values, impurities accumulate and copper alloys form on the tips of the electrodes, altering their resistance value), and therefore the electrodes must be dressed.

Dressing is a process of periodic revitalization of the electrode contact surface (i.e., regeneration of the electrode tips). In the database analysed in the present paper, the electrodes are subjected to dressing every 100 welding points and, after 110 dressings, they are replaced with new ones.

In the next section, data analysis will be made plotting the resistance characteristic curves over the time and comparing the trend of the process curves with that of the learning curves.

The main goal of data analysis is to identify potentially dangerous (i.e. low quality) welding points to be properly checked after production by means of non-destructive ultrasonic tests.

\section{Resistance characteristic curves}

In this work, only resistance characteristic curves will be studied, because the other electric curves present the same behaviour over time; moreover, each resistance characteristic curve refers to one single welding process (impulse) that produces one welding point in the time period of $260 \mathrm{~ms}$.

The main high quality welding indices are: (i) high metal melting speed, (ii) low joint cooling speed. In the following, cases of both good and bad quality welding points will be presented.
A welding process is defined as good quality when the corresponding resistance characteristic curve gives indices that are close to the learning characteristic curves. In this case, a correct value of thermal energy is involved into the welding process, and a correct value of the welding core diameter is obtained. On the other hand, starting from the previous indices, two different types of welding defects can be recognized.

First, a welding is glued if the growth rate of its resistance characteristic curve before melting (melting speed) is much lower than the learning characteristic curves. This is due to the fact that, at the welding point, a much lower amount of thermal energy is been released than the learning one, meaning that the joint is absent or presents a small diameter welding core (i.e. cold welding), causing huge reduction in welding quality.

Second, a welding is squeezed if the degrowth rate of its resistance characteristic curve after melting (cooling speed) is much higher than the learning characteristic curves. At the welding point, if much higher amount of thermal energy has been released than the learning one, which involves the presence of welding material expulsion (i.e. hot welding).and a sharp drop in the characteristic curve, therefore causing a strong reduction in the quality of the welding itself.

\subsection{Learning characteristic curves}

In Figure 1, the five different learning resistance characteristic curves of the learning database as a function of the welding time are plotted. Since the quality of the welding points corresponding to these five characteristic curves was verified experimentally through non-destructive tests (i.e. not glued and not squeezed welding points), then these curves will serve as a reference to analyse the quality of the remaining welding points. In particular, starting from the five curves of Figure 1 , the learning maximum, minimum and average resistance characteristic curves are found, which will be used in the following comparisons.

\subsection{Process characteristic curves}

In this section, as an example, two different resistance characteristic curves that belong to the process database are compared with the learning maximum, minimum and average resistance characteristic curves previously obtained.

In Figure 2, the resistance characteristic curve corresponding to the welding point number 750 belonging to the dressing number 7 is presented. This process characteristic curve denotes a good quality of the corresponding welding point, since the welding is both not glued and not squeezed (the values of the melting and cooling speed are very close to the ones of the learning curves). 


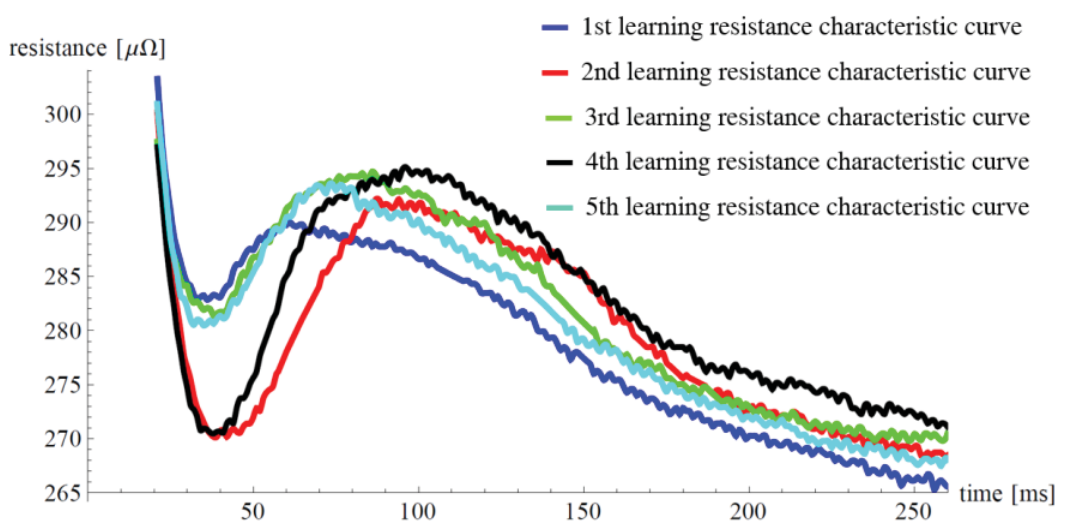

Fig. 1. Plot of the five learning resistance characteristic curves available as a function of the welding time.

In Figure 3, the resistance characteristic curve corresponding to the welding point number 718 belonging to the dressing number 7 is displayed.

Conversely, this process characteristic curve means a low quality of the related welding point. In particular, the welding results both glued (the growth rate of the process curve before melting is much lower than the learning curves, the curve is almost flat at the melting point and the melting point itself is not observable) and squeezed (the degrowth rate of the process curve after melting is much higher than the learning curves, a sharp drop due to welding material expulsion appears).

Following the same procedure of Figures 2-3, the quality of all the individual welding points can be monitored and therefore, more generally, the RSW process reliability can be verified.

Moreover, in order to investigate the trend of the quality of the different welding points within a specific dressing, as an example, in Figure 4 the process resistance characteristic curves that are related to an increasing number of welding points within the dressing number 7 are shown compared with the learning maximum, minimum and average resistance characteristic curves. It can be noted that the first welding point (number 718 ) is both glued and squeezed, the increasing points present a good quality but the last welding points (number 782 and 798) have an increasing difference in the second half of their curves with respect to the learning curves.

By extending the results of Figure 4 to other different cases investigated, it can be stated that the dressed electrodes can reach their maximum efficiency, by regaining their original mechanical properties, only after having carried out at least 20-30 welding points (before, the welding is not good because the correct heat quantity to have a correct melting in the welding point has not yet been reached), e.g. 18 points in the present case.

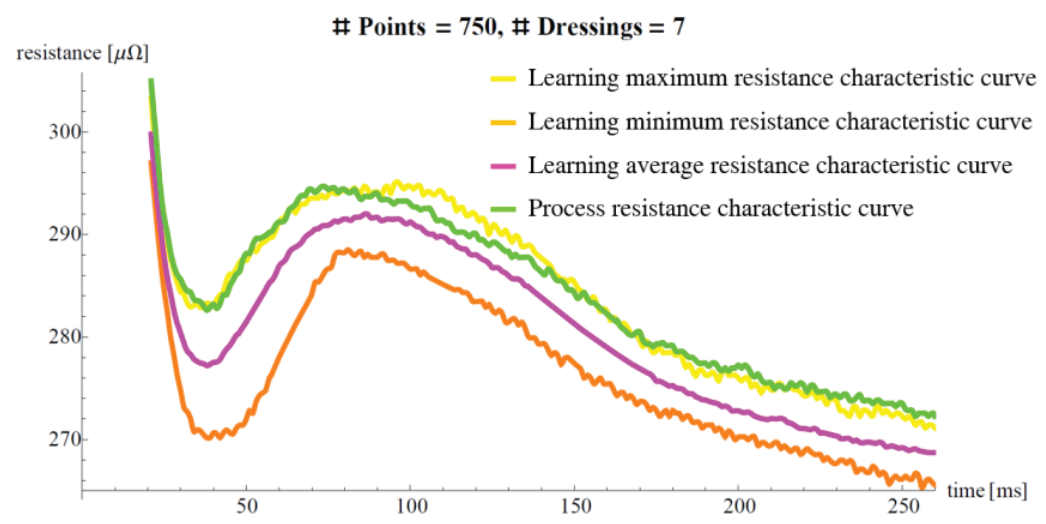

Fig. 2. Plot of the learning maximum, minimum, average and generic process resistance characteristic curves in the time. 


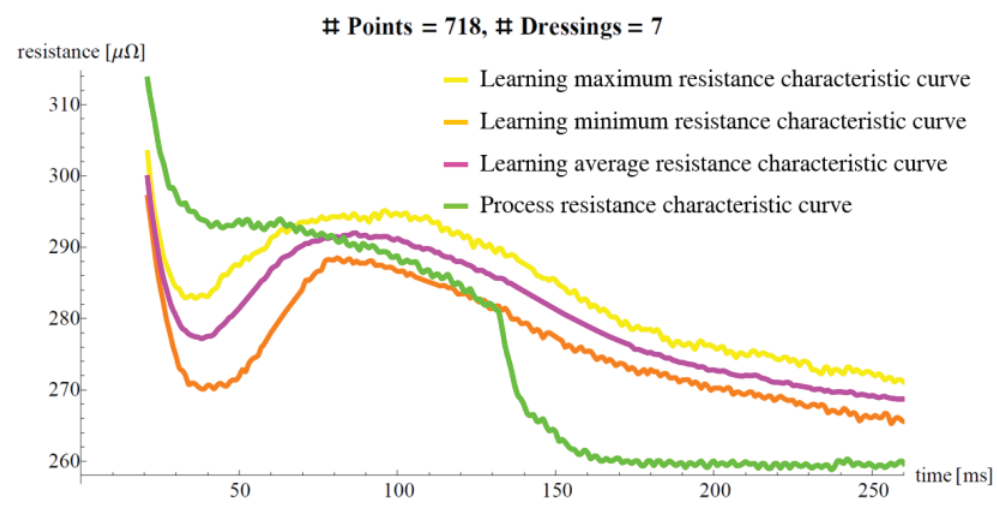

Fig. 3. Plot of the learning maximum, minimum, average and generic process resistance characteristic curves in the time.

\section{Welding point quality}

To more properly identify the quality level of the welding points, in the following, two parameters comparing the generic process with the learning average resistance characteristic curve are used.

\subsection{Residual resistance}

The residual resistance is equal to the difference between the generic process and the learning average resistance characteristic curves at each instant of time during a welding process.

In Figure 5, the residual resistance of the resistance characteristic curve corresponding to the welding point number 750 belonging to the dressing number 7 is displayed together with the residual resistances of the learning maximum and minimum resistance characteristic curves (it is the same case of Figure 2). From this Figure, similarly to Figure 2, it is found that the process residual resistance denotes a good quality of the corresponding welding point (i.e., it is comprised between the learning values with relatively small residual resistance peak in the second half of the curve), therefore the welding is not glued and not squeezed.

In Figure 6, the residual resistance of the resistance characteristic curve corresponding to the welding point number 718 belonging to the dressing number 7 is displayed together with the residual resistances of the learning maximum and minimum resistance characteristic curves (it is the same case of Figure 3). From this Figure, similarly to Figure 3, it is found that the process residual resistance denotes a very low quality of the corresponding welding point (with relatively high residual resistance peaks both in the first and in the second half of the curve), therefore the welding results both glued and squeezed.

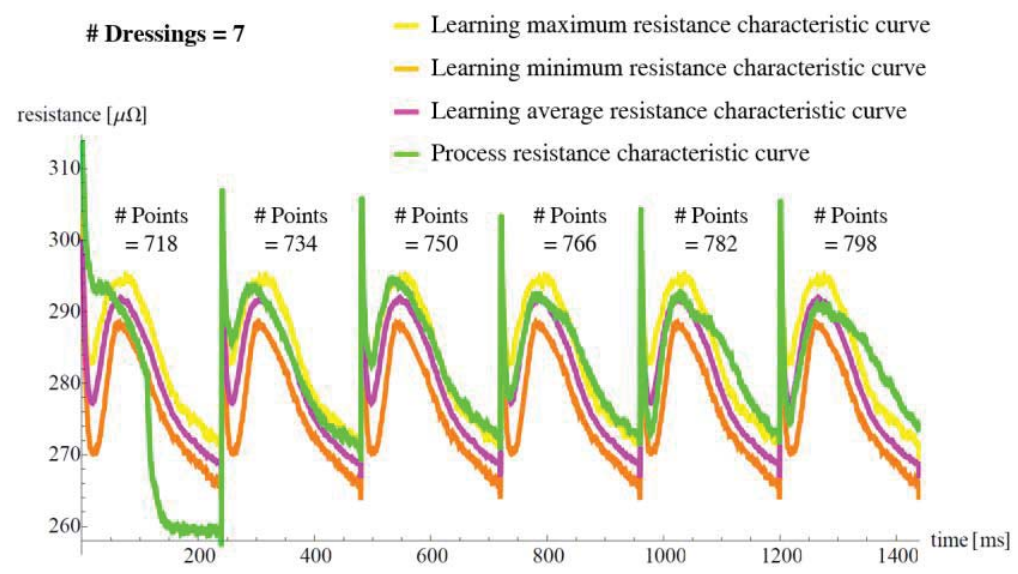

Fig. 4. Plot of the learning maximum, minimum, average and process resistance characteristic curves for the seventh dressing. 


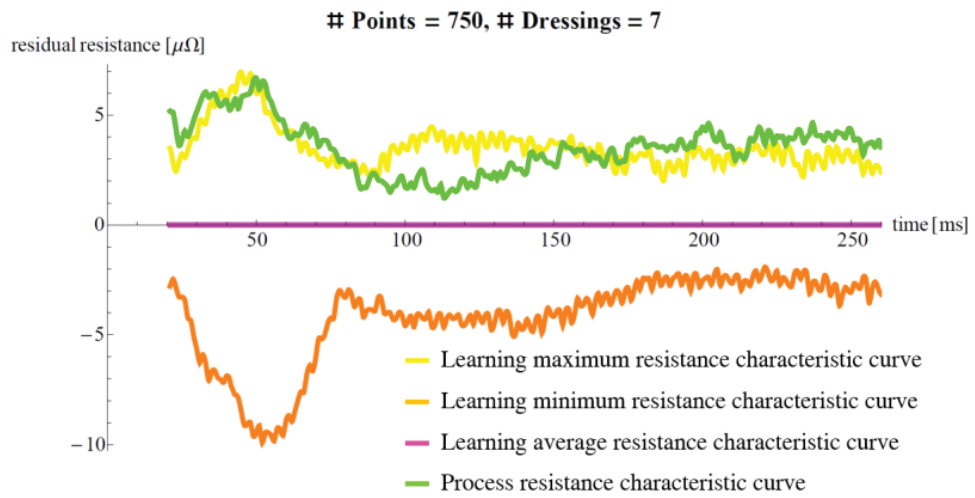

Fig. 5. Residual resistance of the learning maximum, minimum, average and generic process resistance characteristic curves.

Again, to investigate the trend of the quality of different welding points in the same dressing, in Figure 7 the residual resistance characteristic curves that are related to an increasing number of welding points within the dressing number 7 are displayed compared with the learning maximum, and minimum residual resistance characteristic curves. Similarly to Figure 4, it can be noted that the first welding point (number 718) results both glued and squeezed, the increasing points denote good quality but the last welding points (number 782 and 798) have an increasing difference in the second half of the curves with respect to the two learning curves.

Therefore, the residual resistance provides the same results obtained previously by adopting the electrical resistance. In addition, both methods rely on the plot of curves over the welding time period. However, it should be underlined that the quality monitoring based on residual resistance is more rigorous because it considers a relative (i.e. residual) and not an absolute value.

\subsection{Euclidean distance}

The Euclidean distance is the geometric distance between a generic resistance characteristic curve and the learning average resistance characteristic curve at each time instant of a welding process.

It must be stressed that the Euclidean distance parameter was introduced in order to avoid the process curve crossing the learning curves and to synthesize a whole curve with only a point.

In Figure 8, the value of Euclidean distance of the resistance characteristic curve corresponding to the welding point number 750 belonging to the dressing number 7 is displayed together with the values of Euclidean distance of the learning maximum and minimum resistance characteristic curves (same case of Figures 2,5). As in Figures 2 and 5, the process Euclidean distance denotes a good quality of the corresponding welding point (it is lower than the highest learning Euclidean distance, i.e. the one of the minimum resistance curve), which is not glued and not squeezed.

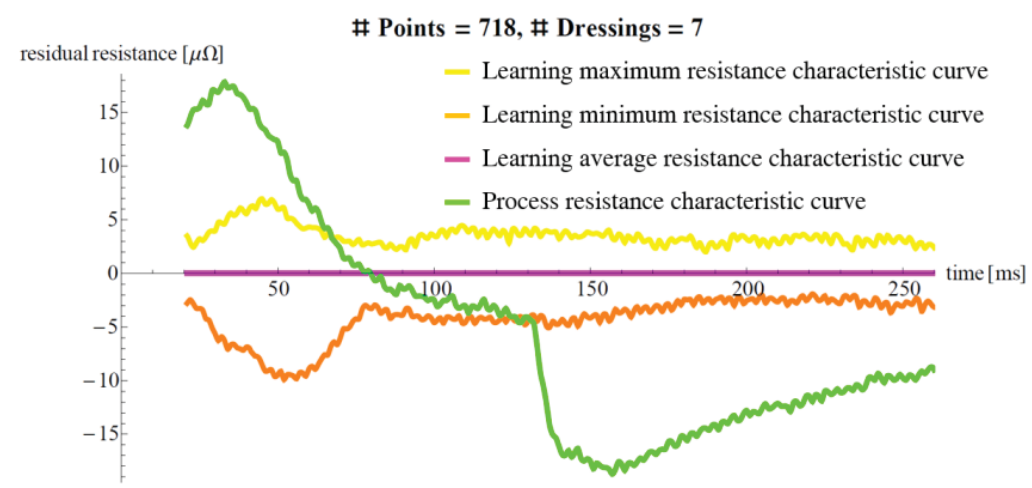

Fig. 6. Residual resistance of the learning maximum, minimum, average and generic process resistance characteristic curves. 


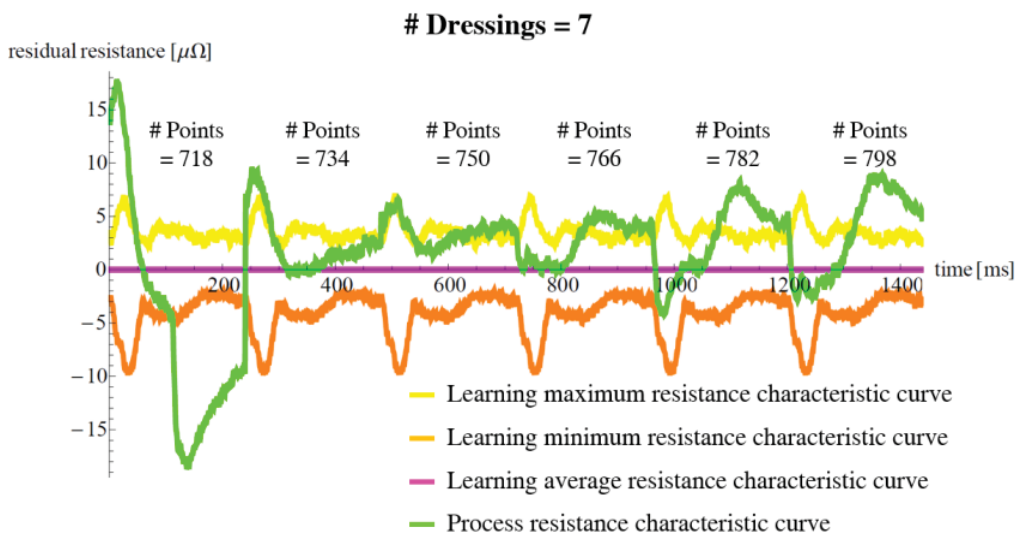

Fig. 7. Residual resistance of the learning maximum, minimum, average and process resistance curves. Seventh dressing.

In Figure 9, the value of Euclidean distance of the resistance characteristic curve corresponding to the welding point number 718 belonging to the dressing number 7 is displayed together with the values of Euclidean distance of the learning maximum and minimum resistance characteristic curves (same case of Figures 3,6). As in Figures 3 and 6, the process Euclidean distance, resulting much higher than both the learning Euclidean distances, denotes very low quality of the related welding point, therefore the welding is defective. However, using the Euclidean distance, unlike electric and residual resistance, it is not possible to properly detect the actual nature of the defect (the welding could be glued, squeezed or both).

Again, to analyse the quality trend of different welding points with the same dressing, in Figure 10 the Euclidean distance of process resistance characteristic curves corresponding to increasing number of welding points with dressing number 7 are displayed in comparison with the Euclidean distance of the learning maximum and minimum resistance characteristic curves. Similarly to the previous findings the first welding point number 718 ) is strongly defective, from the second to the fourth point the welding quality results good and quasi-constant, the Euclidean distance increases, the welding quality of the fifth point (number 782) is sufficiently good while the last welding point (number 798) is slightly defective.

It was obtained that the Euclidean distance provides results very similar to the ones derived previously by adopting the electrical and residual resistance. However, while the last two methods rely on the plot of a curve in the entire welding time, the Euclidean distance is based on the plot of a single point that corresponds to a specific welding impulse. This can be an advantage in the quality monitoring of the welding process, since the quality trend is described using a sequence of points and not of curves, but, as previously said, it is not possible by using the Euclidean distance to detect the actual welding defect.

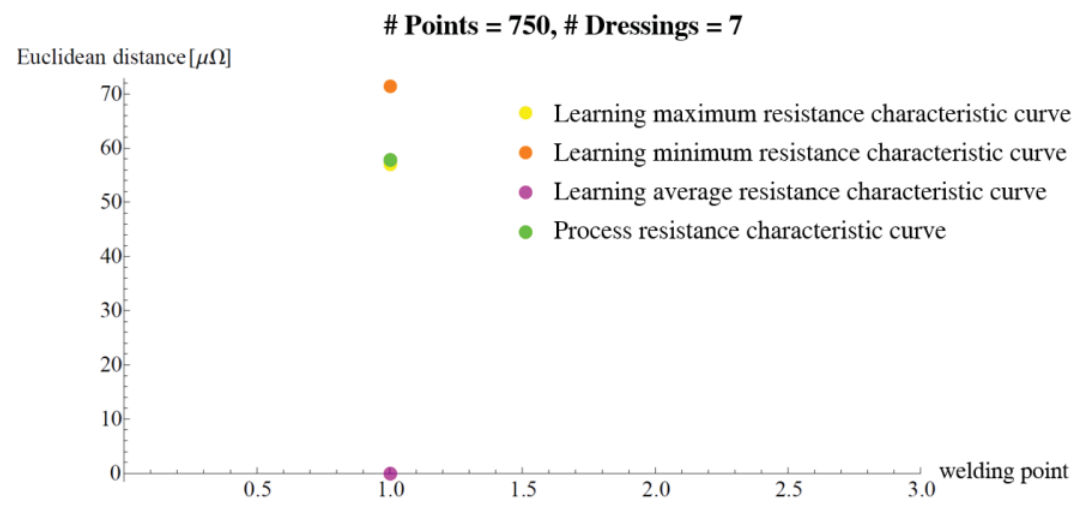

Fig. 8. Euclidean distance of the learning maximum, minimum, average and generic process resistance characteristic curves. 


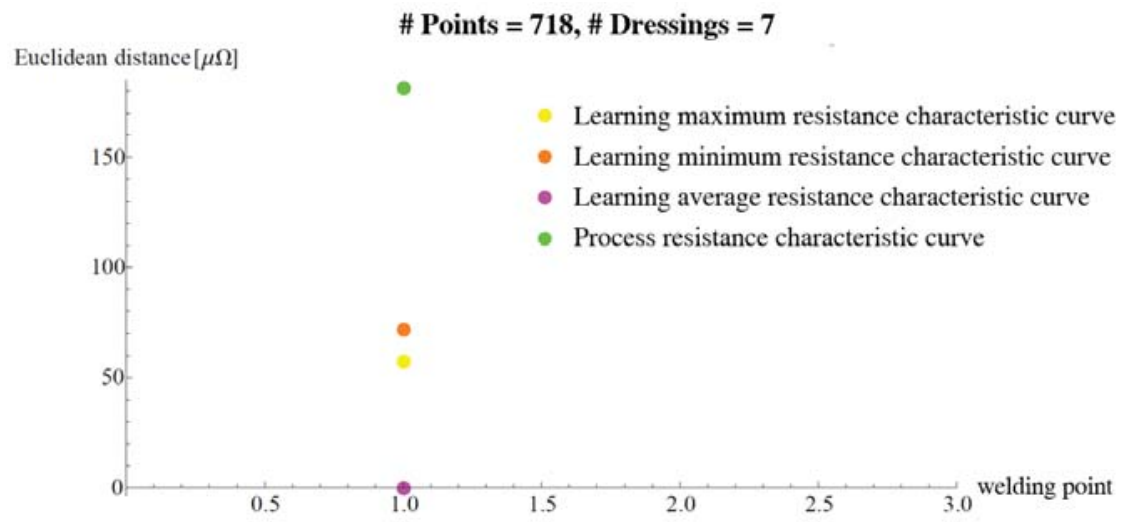

Fig. 9. Euclidean distance of the learning maximum, minimum, average and generic process resistance characteristic curves.

\section{Conclusions}

The goal of the present work is to investigate the reliability of a specific RSW process monitoring the quality of the corresponding welding points.

Each welding point is uniquely represented by a specific resistance characteristic curve in time and a database, containing learning and process resistance characteristic curves, is available.

Different data analysis techniques were used to detect the quality level of each welding point, i.e., resistance, residual resistance and Euclidean distance.

Both good quality and defective (i.e., glued or squeezed) welding points were detected taking as a reference curve the learning average resistance characteristic one.

Moreover, the quality trend of welding points as their number increases for a welding electrode with fixed number of dressings was investigated.

Among all, Euclidean distance represents the most rigorous data analysis technique, because it allows to compare together points and not curves avoiding mutual crossing of curves themselves.

However, unlike the other adopted techniques, Euclidean distance, even if it allows to correctly distinguish a good quality welding point from a defective one, still does not allow to identify the specific nature of the defect itself.

The present study of data analysis techniques for monitoring the quality of welding points will have two future developments:

1. implementation of an optimization law of the dressing process (i.e. maximum number of welding points before each dressing) and of the electrode replacing (i.e. maximum number of dressings before electrode replacement);

2. development and application of advanced expert systems (artificial neural networks) for quality detection of welding points (starting from learning resistance characteristic curves as an input for the experience-based system).

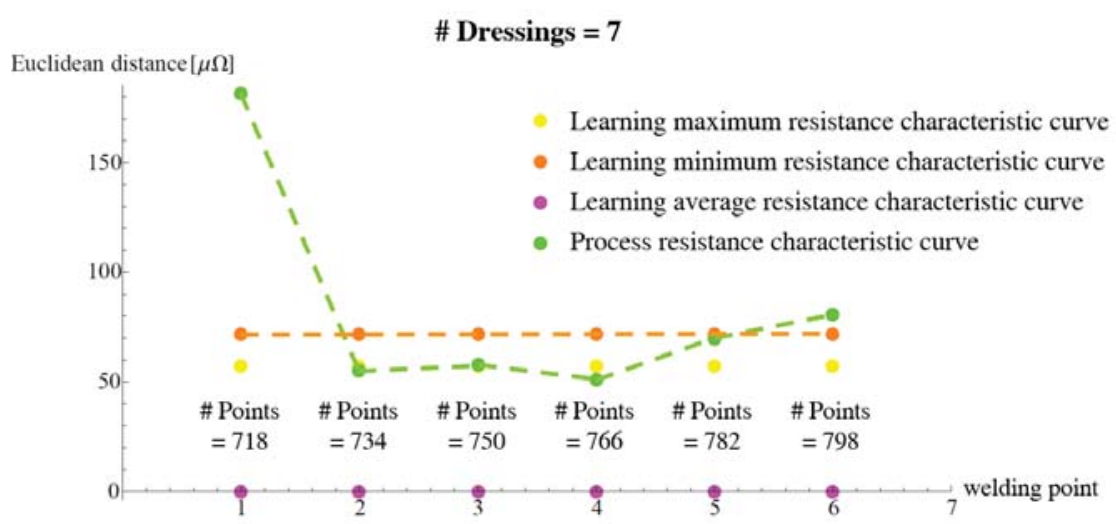

Fig. 10. Euclidean distance of the learning maximum, minimum, average and process resistance curves for the seventh dressing. 


\section{References}

Ambroziak, A., and Korzeniowski, M. (2010). Using resistance spot welding for joining aluminum elements in automotive industry. Archives of Civil and Mechanical Engineering 10, 5-13.

Dhawale, P.A., Kulkarni, M.L. (2017). Electric Resistance Spot Welding: A State of Art. International Journal of Engineering Research \& Technology 5, 1-6.

Jou, M. (2001). Experimental investigation of resistance spot welding for sheet metals used in automotive industry. JSME International Journal Series C 44, 544-552.

Kashiyama, G., and Murakawa, H. (2013). Simulation of nugget formation process in spot welding with process tape. Proceedings of the 1st International Joint Symposium on Joining and Welding, Osaka, Japan, 6-8 November.

Mallaradhya, H., Vijay, K.M., Ranganatha, R., Darshan, S. (2018). Resistance Spot Welding: A Review.
International Journal of Mechanical and Production Engineering Research and Development 8, 403-418.

Mallick, P.K. (2010). Materials, Design and Manufacturing for Lightweight Vehicles. Woodhead Publishing Series in Composites Science and Engineering.

Manladan, S.M., Abdullahi, I., and Hamza, M.F. (2015). A review on the application of resistance spot welding of automotive sheets. Journal of Engineering and Technology 10, 20-37.

Pouranvari, M., and Marashi, S. (2013). Critical review of automotive steels spot welding: process, structure and properties. Science and Technology of welding and joining 185, 361-403.

Tumuluru, M. (2010). Failure Mechanisms of Advanced Welding Processes. Woodhead Publishing Series in Welding and Other Joining Technologies. 